\title{
Fault Tolerant Wireless Mesh Network: An Approach
}

\author{
Jyoti Gupta \\ Assistant Professor \\ IIMT Engineering College
}

\author{
Paramjeet Kaur Bedi \\ Assistant Professor \\ IIMT Engineering College
}

\author{
Nitin Gupta \\ AssistantProfessor \\ HRIT, Ghaziabad
}

\begin{abstract}
Wireless mesh networks (WMNs) have achieved significant development because of fast deployment, easy maintenance and low investment compared with traditional wireless networks. Since WMNs are typically used as wireless backbones, they have the nature that the wireless communication is not constant. Hence, it is important to defend them against link or node failures. In this paper we present an innovative algorithm for fault-tolerant base station planning using antenna arrays in the backbone networks. The algorithm first detects the node's as well as link failure's and then rectifies them using our proposed algorithm. Our paper propose a solution for building a fault tolerant network that can tolerate up to $\mathrm{n}$ faults where $\mathrm{n}$ is the number of antenna arrays used.
\end{abstract}

\section{Keywords}

Wireless Mesh Networks (WMN), fault tolerance, link failure, base station failure, antenna array.

\section{INTRODUCTION}

Wireless Mesh Networks are the infrastructure networks with a multi-hop wireless backbone [2]. Wireless mesh networks (WMNs) consist of mesh routers and mesh clients. The mesh routers have insignificant mobility so they are able to form the backbone of WMNs. They serve network access for both mesh and conventional clients. The Mesh clients can be either stationary or mobile. A WMN is dynamically self-organized and self-configured, with the nodes in the network automatically ascertaining and maintaining mesh connectivity among themselves. WMNs are projected to resolve the restrictions of ad hoc networks, wireless local area networks (WLANs), for improving the throughput and performance of wireless personal area networks (WPANs), and wireless metropolitan area networks (WMANs).

The magnitude of Wireless Mesh networks is not only for providing services like voice/video communication or Internet access at far off places without using any cables, but it also works for other kind of networking solutions[1]. It is a promising technology to connect the worldwide communication with cost effective mechanism and an approach for improving automation processes.

The structure [3] of WMN consists of base stations, distribution system (backbone), links and the mobile stations. The issue is to manage the radio coverage [4] in between all the nodes whether they are base stations or mobile nodes. The need for the system is to achieve the stability or fault tolerance [5] in radio coverage failures. There are two major steps in making a fault tolerant system; the first is the detection of presence of faults in WMN by various ways [6]. This is an important aspect because for making a fault tolerant WMN we need to find that where that faults has occurred. Thze second is to make the radio coverage planning in availability of faults. In our paper we provide an optimal solution for dealing with base station failure and link failure. Our approach deals with the $\mathrm{x}$ number of faults in the backbone.

We have divided our paper in following parts: The first part was the introduction. The second part includes the basics of radio coverage in $\mathrm{WMN}$ with the problem definition of base station planning. The third part contains the related work. In the fourth part, we describe our proposed approach for dealing with the failures in WMN. The fifth part contains the conclusion and future work.

\section{PROBLEM DEFINITION}

\subsection{Radio coverage and Fault Tolerance in respect of WMN}

The WMN infrastructure backbone [6] can be made-up with the use of a collection of radio technologies. The faults can be there in the backbone networks or in the client links. We have to manage both i.e. the link failures and base station failure. The backbone of WMN is formed by connecting the base stations together. The connection between the base stations forms a mesh that helps us to manage the nodes moving from one base station coverage area to another base station coverage area. The mobile nodes or clients are linked with these base stations to form a network among each other. The base station has to maintain the links in between them as well as with the client nodes. There is radio coverage among these nodes and base stations to construct a network for providing communication.

Radio coverage should focus on both: last mile coverage and backbone coverage [7]. Here last mile coverage means connectivity to the mobile stations, within a specified area whereas the backbone coverage means the connectivity among the base stations. The backbone coverage in conventional systems was wired so can be easily managed but in WMN it is wireless so we have to take care of faults in base station coverage too.

There can be two categories of failures in WMN. The first is link failure in which the nodes will move out of the coverage area and there is no link left in between the mesh points. Link failure [5] can be categorized into two classes in requisites of the number of broken links: single-link failure and multiplelink failure. There are three approaches for recovering link 
failures: protection schemes, restoration schemes and hybrid schemes. In the protection scheme, two (or more) link-disjoint paths are chosen a source and a destination node. The source node forwards the data on all of the selected paths. If the link is broken at any point in the path, the destination can still collect the data by some other path. In Restoration schemes, if a failure is detected, it switches the failed path to a backup path dynamically by sharing the backup route but it includes some delay in the recovery process. Hybrid schemes resort to restoration when the protection fails.

Link failure occurs frequently in comparison of node failure and node failure always contains multiple link failures since all links will fail on a failed node. Node failure can results the loss of connection between the various base stations and mobile nodes.

\subsection{Proble m Definition}

For improving the fault tolerance [8], we need to focus on both of the areas: the backbone coverage and last mile coverage. For achieving this we need to determine the following information:

i. Defining the service area coverage. It signifies the area in which we have to provide the network connectivity among different nodes. It helps us to judge the connectivity solutions.

ii. Selection of sites for base stations planning, where the setup of base stations can be done.

iii. Maintaining the radio coverage information:

\section{a. Backbone information}

b. Last mile information

After determining this information we have to evaluate that this radio coverage is correct and fault-tolerant.

The fault tolerance includes two major areas: last mile coverage fault tolerance and backbone coverage fault tolerance [8]. For achieving a fault tolerant $\mathrm{WMN}$, we need such a backbone coverage that can deal with n number of faults.

For dealing with $\mathrm{n}$ number of faults we are using antenna arrays at the base stations. The use of an antenna array can increase the efficiency of wireless communications systems. An array works on the principle that the desired signal and unwanted co channel interferences appear from different directions. The beam pattern of the array is adjusted to suit the requirements by combining signals from different antennas with appropriate weighting.

By using array of antenna at base station, we can extend the range and coverage. When the base station coverage is increased by antenna arrays, we can increase the coverage range in back up.

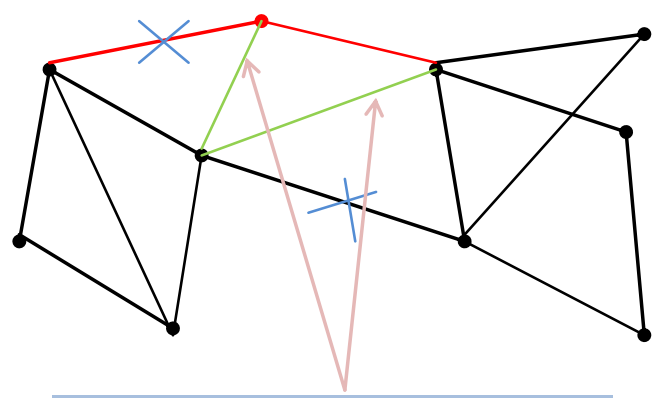

New Links by antenna arrays

Fig. 1: Creation of new links by antenna arrays if more than one link are broken

Our approach provides the solution for dealing the backbone coverage faults which covers the link failures and node failures. If we can recover from fault if any of BS fails, the network can be managed without breaking off the connectivity within the network nodes may be base stations or mobile nodes.

\section{RELATED WORK}

Engineering automation networks have classically been inaccessible single-cell networks or classic infrastructure networks with multiple cells. This means that coverage set up is obligatory only for the 'last mile', i.e. the connection between a base station and a mobile station, e.g. [9]. In multihop wireless mesh networks, the coverage planning of the backbone network is not focused much. Maximum researchers on radio network planning think about network throughput as a main planning goal, e.g. [4]. The need of the hour in the requirement of engineering networks is availability.

As the new technologies are coming into the market (e.g. Zigbee, Wireless HART), the challenge is to make base station planning. Some of the papers [10] are presenting confronts for developing a planning tool for wireless sensor networks and also signifies that base station planning is an important requirement. But still a systematic approach is missing in planning multi-hop wireless networks with respect to fault tolerance requirements of industrial automation networks.

The previously defined algorithms [8, 11] had designed backbone coverage with minimum number of base stations in such a way that the end-to-end throughput requirements for Internet access in areas with no alternative high-speed wired connection can be done. These approaches lead to the connected backbone graph but do not provide fault tolerance.

One of the researchers [12] provides the solution for the fault tolerance in wireless ad-hoc network. It shows a scenario for determining the probability that a backbone network graph is $\mathrm{k}$-connected, based on the transmission range. But the basic assumption of the method is that the network can be modeled as a union disk graph, where all nodes within a given transmission range are perfectly reachable and all nodes outside this range are not reachable at all. It has been shown that this network model in the general case does not comply with real networks. 
Many papers cover the problem of fault-tolerant communication in mobile multi-hop ad-hoc networks (MANET) and in wireless sensors networks. Papers allowing for fault-tolerant routing, for instance [13], have as a prerequisite at least biconnected backbone network, but still the base station planning problem is out of their scope.

S. Ivanov et al papers [7] approach is to extend the existing methods from infrastructure network planning to plan the multi-hop wireless mesh networks with fault-tolerance aspects. The base station planning algorithm provides coverage in a predefined area by adjusting the number and the position of the base stations. But this approach also works for limited number of faults that is can work only for one base station failure.

\section{PROPOSED WORK}

Our approach deals with the mesh network consisting of $n$ nodes. There will be a graph of the connected base stations where BS can be considered as nodes and the links between the nodes will work like the edges in the graph. There is a property bi connectivity in a network that can be identified by the minimum degree of the network. We can get the minimum degree of all the nodes for this graph. The minimum degree of the graph is the number of incoming and outgoing links of the node. In graph theory, the minimum degree is a necessary but not sufficient condition for k-connectivity [14]. This means that a k-connected graph has a minimum degree of $\mathrm{k}$, but a graph with minimum degree of $\mathrm{k}$ is not necessary $\mathrm{k}$-connected. Formally, this rule applies to the backbone of wireless mesh networks. However, radio coverage in $\mathrm{WMN}$ has two aspects: backbone and last mile.

For covering last mile fault tolerance it is necessary that each node should be covered by at least two base stations. So we check that there is a minimum degree of 2 maintained in the resultant graph. We will divide our approach into following parts to cover backbone fault tolerance.

\subsection{Identify the Degree of each node}

According to our approach, the first step we will take is to identify the degree of each node in the graph. Each node should have a minimu $m$ degree of 3 .

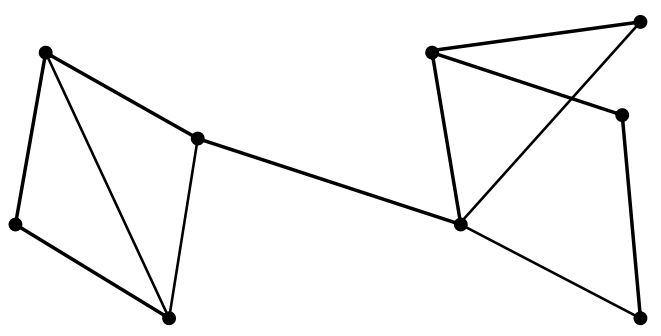

Fig. 2: Graph of $\mathrm{n}$ base stations with their connectivity

\subsection{Segregation of graph}

After identification of degree of nodes, we will remove each edge from the graph and check whether after removing the edge, the graph is still connected or not. And if the graph is disconnected, by removing any edge then we divide the graph into two.

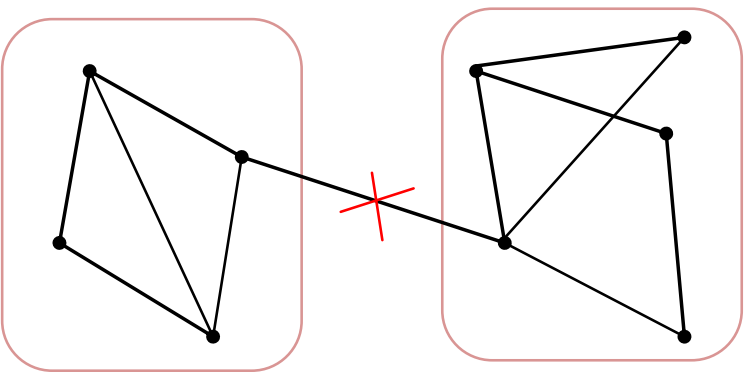

Fig. 3: Classification of graph into two disjoint parts after breaking link

\subsection{Base Station Planning}

When our graph shows that there is no link in between those two graphs, we can plan a new base station to maintain the connectivity in between these two graphs.

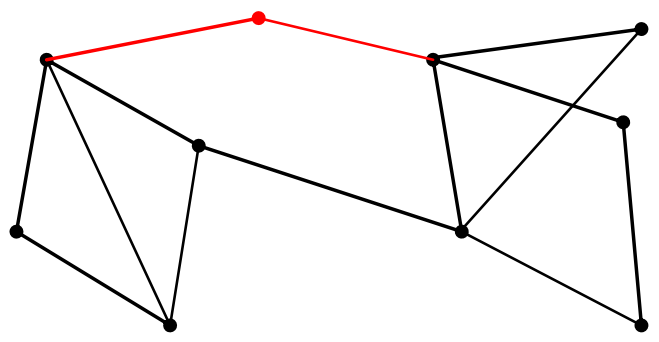

Fig. 4: Placing a new base station for maintaining the link

After planning and establishing the new base stations, our backbone become much fault tolerant but it can work only till one link fails.

\section{ALGORITHM}

1. Find the degree of each node.

2. Identify those nodes having degree less than 3 .

3. For each edge in the graph

a. Remove the ed ge

b. Check the connectivity of the graph

c. If the graph is disconnected then

i. Place a new base station to make the graph connected

d. End if

e. Place back the ed ge just removed

\section{End For}

\section{CONCLUSION AND FUTURE WORK}

The proposed approach for fault-tolerant base station planning in wireless mesh networks manages sufficient amount of fault tolerance in link failures as well as backbone failures in a costeffective manner. It will help to get an efficient and costeffective mechanism for fault tolerant connectivity among 
different base stations and clients. This approach works for a configurable amount of fault tolerance, i.e. the radio coverage remains correct if $\mathrm{k}$ base stations fail and/or 1 links fail.

In future work we will evaluate the effect of the work will be to provide a formal proof of the algorithm. In addition, we will extend the approach for increased radio coverage not as backup plan but as a permanent solution.

\section{REFERENCES}

[1.] Andreas Willig. Recent and Emerging Topics in Wireless Industrial Communications: A Selection. IEEE Transactions on Industrial Informatics, 4:102-121, 2008.

[2.] Ian F. Akyildiz, Xudong Wang, Weilin Wang. Wireless mesh networks: a survey. Elsevier Journal on Computer Networks, 47(445-487), 2005.

[3.] Wireless mesh network. http://en.wikipedia.org/wiki/ Wireless_mesh_network.

[4.] S.Bosio, A. Capone, and M. Cesana. Radio planning of wireless local area networks. IEEE/ACM Transactions on Networking, 15(6): 1414-1427, 2007.

[5.] Dr. Richard A. Frost. A Survey: Fault Tolerance for Link Failure in Wireless Mesh Networks. 60-510 Background Reading and Survey.

[6.] S. Ivanov and E.Nett. Fault tolerant coverage planning in wireless networks. In 27th IEEE International Sy mposium on Reliable Distributed Systems (SRDS 2008), 6-8 October, Napoli, Italy, 2008.

[7.] S. Ivanov, E.Nett. And R. Schumann. Fault-tolerant base station planning of wireless mesh networks in Dynamic Industrial Environments.

[8.] E. Amaldi, A. Capone, M. Cesana, I. Filippini, and F. Malucelli. Optimization models and methods for planning wireless mesh networks. Elsevier Journal on Computer Networks, 52(11):2159-2171, 2008.
[9.] T. M. Chan, K. F. Man, K. S. Tang, and S. Kwong. A jumping-genes paradigm for optimizing factory wlan network. IEEE TRANSACTIONS ON INDUSTRIAL INFORMATICS, 3:33-43, 2007.

[10.]A. Ray. Planning and analysis tool for large scale deployment of wireless sensor network. International Journal of Next-Generation Networks (IJNGN), 1(1):2936, December 2009.

[11.]A. So and B. Liang. Optimal placement and channel assignment of relay stations in heterogeneous wireless mesh networks by modified bender's decomposition. Elsevier Journal on Ad Hoc Networks, 7(1):118-135, 2009.

[12.]X.-Y. Li, P.-J. Wan, Y. Wang, and C.-W. Yi. Fault tolerant deployment and topology control in wireless networks. In MobiHoc '03: Proceedings of the 4th ACM international symposium on Mobile ad hoc networking \& computing, pages 117-128, New York, NY, USA, 2003. ACM.

[13.]D. Avresky and N. Natchev. Dynamic reconfiguration in computer clusters with irregular topologies in the presence of multiple node and link failures. IEEE Transactions on Computers, 54(5):603-615, 2005.

[14.]R. Diestel. Graph Theory. Springer-Verlag, Heidelberg, 2005.

[15.]LAL C. GODARA, Applications of Antenna Arrays to Mobile Communications, Part I: Performance improvement, Feasibility, and System Considerations. PROCEEDINGS OF THE IEEE, VOL. 85, NO. 7, JULY 1997.

[16.]B. Ottersten and P. Zetterberg. Base station Antenna Arrays in Mobile Communications. Proceedings of 7 th Tyrrhenian International Workshop on digital communications. Sep, 1995. 